\title{
INTUITIONISTIC FUZZY ALMOST GENERALIZED SEMI-PRE CONTINUOUS MAPPINGS
}

\author{
R. SANTHI AND D. JAYANTHI
}

\begin{abstract}
In this paper we introduce intuitionistic fuzzy almost generalized semi-pre continuous mappings. We investigate some of its properties. Also we provide the relation between intuitionistic fuzzy almost generalized semi-pre continuous mappings and other intuitionistic fuzzy continuous mappings.
\end{abstract}

\section{Introduction}

After the introduction of fuzzy sets by Zadeh [13], there have been a number of generalizations of this fundamental concept. The notion of intuitionistic fuzzy sets introduced by Atanassov [2] is one among them. Using the notion of intuitionistic fuzzy sets, Coker [5] introduced the notion of intuitionistic fuzzy topological spaces. Intuitionistic fuzzy semi-pre continuous mappings in intuitionistic fuzzy topological spaces are introduced by Young Bae Jun and Seok- Zun Song [12]. R. Santhi and D. Jayanthi [9] introduced the notion of intuitionistic fuzzy generalized semi-pre continuous mappings and intuitionistic fuzzy generalized semipre irresolute mappings. In this paper we introduce intuitionistic fuzzy almost generalized semi-pre continuous mappings. We investigate some of its properties. Also we provide the condition for an intuitionistic fuzzy almost generalized semi-pre continuous mapping to be an intuitionistic fuzzy generalized semi-pre continuous mappings.

\section{Preliminaries}

Definition 2.1. An intuitionistic fuzzy set (IFS in short) $A$ in $X$ is an object having the form

$$
A=\left\{\left\langle x, \mu_{A}(x), v_{A}(x)\right\rangle \mid x \in X\right\}
$$

where the functions $\mu_{A}: X \rightarrow[0,1]$ and $v_{A}: X \rightarrow[0,1]$ denote the degree of membership (namely $\mu_{A}(x)$ ) and the degree of non-membership (namely $v_{A}(x)$ ) of each element $x \in X$ to the set $A$, respectively, and $0 \leq \mu_{A}(x)+v_{A}(x) \leq 1$ for each $x \in X$. Denote by $\operatorname{IFS}(X)$, the set of all intuitionistic fuzzy sets in $X$.

Corresponding author: R. Santhi.

Key words and phrases. Intuitionistic fuzzy topology, intuitionistic fuzzy generalized semi-pre $T_{1 / 2}$ space, intuitionistic fuzzy almost generalized semi-pre continuous mappings. 
Definition 2.2. ([2]) Let $A$ and $B$ be IFSs of the form $A=\left\{\left\langle x, \mu_{A}(x), v_{A}(x)\right\rangle \mid x \in X\right\}$ and $B=$ $\left\{\left\langle x, \mu_{B}(x), v_{B}(x)\right\rangle \mid x \in X\right\}$. Then

(a) $A \subseteq B$ if and only if $\mu_{A}(x) \leq \mu_{B}(x)$ and $v_{A}(x) \geq v_{B}(x)$ for all $x \in X$.

(b) $A=B$ if and only if $A \subseteq B$ and $B \subseteq A$.

(c) $A^{c}=\left\{\left\langle x, v_{A}(x), \mu_{A}(x)\right\rangle \mid x \in X\right\}$

(d) $A \cap B=\left\{\left\langle x, \mu_{A}(x) \wedge \mu_{B}(x), v_{A}(x) \vee v_{B}(x),\right\rangle \mid x \in X\right\}$.

(e) $A \cup B=\left\{\left\langle x, \mu_{A}(x) \vee \mu_{B}(x), v_{A}(x) \wedge v_{B}(x),\right\rangle \mid x \in X\right\}$

For the sake of simplicity, we shall use the notation $A=\left\langle x, \mu_{A}, v_{A}\right\rangle$ instead of $A=\left\{\left\langle x, \mu_{A}(x), v_{A}(x)\right\rangle \mid\right.$ $x \in X\}$.

The intuitionistic fuzzy sets $0_{\sim}=\{\langle x, 0,1\rangle \mid x \in X\}$ and $1_{\sim}=\{\langle x, 1,0\rangle \mid x \in X\}$ are respectively the empty set and the whole set of $X$.

Definition 2.3. ([11]) The IFS $c(\alpha, \beta)=\left\langle x, c_{\alpha}, c_{1-\beta}\right\rangle$ where $\alpha \in(0,1], \beta \in[0,1)$ and $c_{\alpha}+c_{1-\beta} \leq 1$ is called an intuitionistic fuzzy point in $X$.

Note that an intuitionistic fuzzy point $c(\alpha, \beta)$ is said to belong to an IFS $A=\left\langle x, \mu_{A}, v_{A}\right\rangle$ of $X$ denoted by $c(\alpha, \beta) \in A$ if $\alpha \leq \mu_{A}$ and $\beta \geq v_{A}$.

Definition 2.4. ([11]) Two IFSs are said to be $q$-coincident ( $A_{q} B$ in short) if and only if there exists an element $x \in X$ such that $\mu_{A}(x)>v_{B}(x)$ and $v_{A}(x)<\mu_{B}(x)$.

Definition 2.5. ([5]) An intuitionistic fuzzy topology (IFT for short) on $X$ is a family $\tau$ of IFSs in $X$ satisfying the following axioms.

(i) $0 \sim, 1_{\sim} \in \tau$.

(ii) $G_{1} \cap G_{2} \in \tau$ for any $G_{1}, G_{2} \in \tau$.

(iii) $\cup G_{i} \in \tau$ for any family $\left\{G_{i} \mid i \in J\right\} \subseteq \tau$.

In this case the pair $(X, \tau)$ is called an intuitionistic fuzzy topological space (IFTS in short) and any IFS in $\tau$ is known as an intuitionistic fuzzy open set in $X$. The complement $A^{c}$ of an intuitionistic fuzzy open set $A$ in $\operatorname{IFTS}(X, \tau)$ is called an intuitionistic fuzzy closed set in $X$.

Definition 2.6. ([5]) Let $(X, \tau)$ be an IFTS and $A=\left\langle x, \mu_{A}, v_{A}\right\rangle$ be an IFS in $X$. Then the intuitionistic fuzzy interior and intuitionistic fuzzy closure are defined by

$$
\begin{aligned}
\operatorname{int}(A) & =\bigcup\{G \mid G \text { is an intuitionistic fuzzy open set in } X \text { and } G \subseteq A\} \\
\operatorname{cl}(A) & =\bigcap\{K \mid K \text { is an intuitionistic fuzzy closed set in } X \text { and } A \subseteq K\} .
\end{aligned}
$$

Note that for any IFS $A$ in $(X, \tau)$, we have $\operatorname{cl}\left(A^{c}\right)=[\operatorname{int}(A)]^{c}$ and $\operatorname{int}\left(A^{c}\right)=[\operatorname{cl}(A)]^{c}[12]$. 
Definition 2.7. ([7]) An IFS $A=\left\langle x, \mu_{A}, v_{A}\right\rangle$ in an IFTS $(X, \tau)$ is said to be an

(i) intuitionistic fuzzy semi closed set if $\operatorname{int}(\operatorname{cl}(A)) \subseteq A$.

(ii) intuitionistic fuzzy semi closed set if $\operatorname{cl}(\operatorname{int}(A)) \subseteq A$.

(iii) intuitionistic fuzzy semi closed set if $\operatorname{cl}(\operatorname{int}(\operatorname{cl}(A))) \subseteq A$.

The respective complements of the above intuitionistic fuzzy closed sets are called their respective intuitionistic fuzzy open sets.

The family of all intuitionistic fuzzy semi closed sets, intuitionistic fuzzy pre closed sets, and intuitionistic fuzzy $\alpha$ closed sets (respectively intuitionistic fuzzy semi open sets, intuitionistic fuzzy pre open sets and intuitionistic fuzzy $\alpha$ open sets) of an IFTS $(X, \tau)$ are respectively denoted by $\operatorname{IFSC}(X), \operatorname{IFPC}(X)$ and $\operatorname{IF} \alpha \mathrm{C}(X)$ (respectively $\operatorname{IFSO}(X), \operatorname{IFPO}(X)$ and $\operatorname{IF} \alpha \mathrm{O}(X))$.

Definition 2.8. ([12]) An IFS $A=\left\langle x, \mu_{A}, v_{A}\right\rangle$ in an $\operatorname{IFTS}(X, \tau)$ is said to be an

(i) intuitionistic fuzzy semi closed set if there exists an intuitionistic fuzzy pre closed set $B$ such that int $(B) \subseteq A \subseteq B$.

(ii) intuitionistic fuzzy semi-pre open set if there exists an intuitionistic fuzzy pre open set $B$ such that $B \subseteq A \subseteq \operatorname{cl}(B)$.

The family of all intuitionistic fuzzy semi-pre closed sets (respectively intuitionistic fuzzy semi-pre open sets) of an $\operatorname{IFTS}(X, \tau)$ is denoted by $\operatorname{IFSPC}(X)$ (respectively $\operatorname{IFSPO}(X)$ ).

Every intuitionistic fuzzy semi closed set (respectively intuitionistic fuzzy semi open set) and every intuitionistic fuzzy pre closed set (respectively intuitionistic fuzzy pre open set) is an intuitionistic fuzzy semi-pre closed set (respectively intuitionistic fuzzy semi-pre open set). But the separate converses need not be true in general [12].

Note that an IFS $A$ is an intuitionistic fuzzy semi-pre closed set if and only if int $(\operatorname{cl}(\operatorname{int}(A)))$ $\subseteq A$ and an intuitionistic fuzzy semi-pre open set if and only if $A \subseteq \operatorname{cl}(\operatorname{int}(\operatorname{cl}(A)))$ [8].

Definition 2.9. ([8]) Let $A$ be an IFS in an IFTS $(X, \tau)$. Then the semi-pre interior and the semi- pre closure of $A$ are defined by

$$
\begin{aligned}
\operatorname{spint}(A) & =\bigcup\{G \mid G \text { is an intuitionistic fuzzy semi-pre open set in } X \text { and } G \subseteq A\} . \\
\operatorname{spcl}(A) & =\bigcap\{K \mid K \text { is an intuitionistic fuzzy semi-pre closed set in } X \text { and } A \subseteq K\} .
\end{aligned}
$$

Note that for any IFS $A$ in $(X, \tau)$, we have $\operatorname{spcl}\left(A^{c}\right)=[\operatorname{spint}(A)]^{c}$ and $\operatorname{spint}\left(A^{c}\right)=[\operatorname{spcl}(A)]^{c}[8]$.

Definition 2.10. ([11]) An IFS $A$ ia an

(i) intuitionistic fuzzy regular closed set if $A=\operatorname{cl}(\operatorname{int}(A))$. 
(ii) intuitionistic fuzzy generalized closed set if $\operatorname{cl}(A) \subseteq U$ whenever $A \subseteq U$ and $U$ is an intuitionistic fuzzy open set.

(iii) intuitionistic fuzzy regular generalized closed set if $\operatorname{cl}(A) \subseteq U$ whenever $A \subseteq U$ and $U$ is an intuitionistic fuzzy regular open set.

The family of all intuitionistic fuzzy regular closed sets and intuitionistic fuzzy generalized closed sets (respectively intuitionistic fuzzy regular open sets and intuitionistic fuzzy generalized open sets) of an $\operatorname{IFTS}(X, \tau)$ are respectively denoted by $\operatorname{IFRC}(X)$ and $\operatorname{IFGC}(X)$ (respectively $\operatorname{IFRO}(X)$ and $\operatorname{IFGO}(X)$ ).

Definition 2.11. ([8]) An IFS $A$ ia an IFTS $(X, \tau)$ is said to be an intuitionistic fuzzy generalized semi-pre closed set if $\operatorname{spcl}(A) \subseteq U$ whenever $A \subseteq U$ and $U$ is an intuitionistic fuzzy open set in $(X, \tau)$.

Every intuitionistic fuzzy closed set, intuitionistic fuzzy generalized closed set, intuitionistic fuzzy semi closed set, intuitionistic fuzzy pre closed set, intuitionistic fuzzy regular closed set, intuitionistic fuzzy $\alpha$ closed set and intuitionistic fuzzy semi-pre closed set is an intuitionistic fuzzy generalized semi-pre closed set but the separate converses may not be true in general [9].

The family of all intuitionistic fuzzy generalized semi-pre closed sets of an $\operatorname{IFTS}(X, \tau)$ is denoted by $\operatorname{IFGSPC}(X)$.

Definition 2.12. ([8]) Let $A$ be an IFS in an IFTS $(X, \tau)$. Then the generalized semi-pre interior and the generalized semi- pre closure of $A$ are defined by

$\operatorname{gspint}(A)=\bigcup\{G \mid G$ is an intuitionistic fuzzy generalized semi-pre open set in $X$ and $G \subseteq A\}$. $\operatorname{gspcl}(A)=\bigcap\{K \mid K$ is an intuitionistic fuzzy generalized semi-pre closed set in $X$ and $A \subseteq K\}$.

Note that for any IFS $A$ in $(X, \tau)$, we have $\operatorname{gspcl}\left(A^{c}\right)=[\operatorname{gspint}(A)]^{c}$ and $\operatorname{gspint}\left(A^{c}\right)=[\operatorname{gspcl}(A)]^{c}$.

Remark 2.13. If an IFS $A$ in an IFTS $(X, \tau)$ is an intuitionistic fuzzy generalized semipre closed set in $X$, then $\operatorname{gspcl}(A)=A$. But the converse may not be true in general, since intersection does not exist in intuitionistic fuzzy generalized semi-pre closed sets [9].

Remark 2.14. If an IFS $A$ in an IFTS $(X, \tau)$ is an intuitionistic fuzzy generalized semipre open set in $X$, then gspint $(A)=A$. But the converse may not be true in general, since union does not exist in intuitionistic fuzzy generalized semi-pre open sets [9].

Definition 2.15. ([8]) The complement $A^{c}$ of an intuitionistic fuzzy generalized semi-pre closed set $A$ in an IFTS $(X, \tau)$ is called an intuitionistic fuzzy generalized semi-pre open set in $X$. 
Every intuitionistic fuzzy open set, intuitionistic fuzzy generalized open set, intuitionistic fuzzy semi open set, intuitionistic fuzzy pre open set, intuitionistic fuzzy regular open set, intuitionistic fuzzy $\alpha$ open set and intuitionistic fuzzy semi-pre open set is an intuitionistic fuzzy generalized semi-pre open set but the separate converses may not be true in general [8].

The family of all intuitionistic fuzzy generalized semi-pre open sets of an $\operatorname{IFTS}(X, \tau)$ is denoted by $\operatorname{IFGSPO}(X)$.

Definition 2.16. ([7]) Let $f$ be a mapping from an IFTS $(X, \tau)$ into an IFTS $(Y, \sigma)$. Then $f$ is said to be an intuitionistic fuzzy continuous mapping if $f^{-1}(B) \in \operatorname{IFO}(X)$ for every $B \in \sigma$.

Definition 2.17. ([3]) Let $f$ be a mapping from an $\operatorname{IFTS}(X, \tau)$ into an IFTS $(Y, \sigma)$. Then $f$ is said to be an

(i) intuitionistic fuzzy semi continuous mapping (IFSCts. for short) if $f^{-1}(B) \in \operatorname{IFSO}(X)$ for every $B \in \sigma$.

(ii) intuitionistic fuzzy $\alpha$-continuous mapping (IF $\alpha$ Cts. for short) if $f^{-1}(B) \in \operatorname{IF} \alpha \mathrm{O}(X)$ for every $B \in \sigma$.

(iii) intuitionistic fuzzy pre continuous mapping (IFPCts. for short) if $f^{-1}(B) \in \operatorname{IFPO}(X)$ for every $B \in \sigma$.

Every intuitionistic fuzzy continuous mapping is an intuitionistic fuzzy $\alpha$ continuous mapping and every intuitionistic fuzzy $\alpha$ continuous mapping is an intuitionistic fuzzy semi continuous mapping as well as an intuitionistic fuzzy pre continuous mapping, but the separate converses may not be true in general [7].

Definition 2.18. ([10]) Let $c(\alpha, \beta)$ be an intuitionistic fuzzy point of an IFTS $(X, \tau)$. An IFS $A$ of $X$ is called an intuitionistic fuzzy neighborhood (IFN for short) of $c(\alpha, \beta)$ if there exists an intuitionistic fuzzy open set $B$ in $X$ such that $c(\alpha, \beta) \in B \subseteq A$.

Definition 2.19. ([11]) Let $f$ be a mapping from an $\operatorname{IFTS}(X, \tau)$ into an $\operatorname{IFTS}(Y, \sigma)$. Then $f$ is said to be an intuitionistic fuzzy generalized continuous mapping (IFGCts. for short) if $f^{-1}(B) \in \operatorname{IFGC}(X)$ for every intuitionistic fuzzy closed set $B$ in $Y$.

Every intuitionistic fuzzy continuous mapping is an intuitionistic fuzzy generalized continuous mapping but the converse may not be true in general [11].

Definition 2.20. ([12]) Let $f$ be a mapping from an $\operatorname{IFTS}(X, \tau)$ into an $\operatorname{IFTS}(Y, \sigma)$. Then $f$ is said to be an intuitionistic fuzzy semi-pre continuous mapping (IFSPCts. for short) if $f^{-1}(B) \in \operatorname{IFSPO}(X)$ for every $B \in \sigma$. 
Every intuitionistic fuzzy semi continuous mapping and intuitionistic fuzzy pre continuous mapping are intuitionistic fuzzy semi-pre continuous mapping but the converses may not be true in general [12].

Definition 2.21. Let $f$ be a mapping from an $\operatorname{IFTS}(X, \tau)$ into an $\operatorname{IFTS}(Y, \sigma)$. Then $f$ is said to be an intuitionistic fuzzy semi-pre irresolute mapping if $f^{-1}(B)$ is an intuitionistic fuzzy semi-pre closed set of $X$ for every intuitionistic fuzzy semi-pre closed set $B$ in $Y$.

Definition 2.22. ([8]) If every intuitionistic fuzzy generalized semi-pre closed set in $(X, \tau)$ is an intuitionistic fuzzy semi-pre closed set in $(X, \tau)$, then the space can be called as an intuitionistic fuzzy semi- pre $\mathscr{T}_{1 / 2}$ space.

Theorem 2.23. For any IFS $A$ in an $\operatorname{IFTS}(X, \tau)$ where $X$ is an intuitionistic fuzzy semipre $T_{1 / 2}$ space, $A \in I F G S P O(X)$ if and only if for every intuitionistic fuzzy point $c(\alpha, \beta) \in A$, there exists an intuitionistic fuzzy generalized semi-pre open set $B$ in $X$ such that $c(\alpha, \beta) \in B \subseteq A$.

Proof. Necessity: If $A \in \operatorname{IFGSPO}(X)$, then we can take $B=A$ so that $c(\alpha, \beta) \in B \subseteq A$ for every intuitionistic fuzzy point $c(\alpha, \beta) \in A$.

Sufficiency: Let $A$ be an IFS in $X$ and assume that there exists $B \in \operatorname{IFGSPO}(X)$ such that $c(\alpha, \beta) \in$ $B \subseteq A$. Since $X$ is an intuitionistic fuzzy semi-pre $T_{1 / 2}$ space, $B$ is an intuitionistic fuzzy semipre open set of $X$. Then $A=\bigcup_{c(\alpha, \beta) \in A}\{c(\alpha, \beta)\} \subseteq \bigcup_{c(\alpha, \beta) \in A} B \subseteq A$. Therefore $A \underset{c(\alpha, \beta) \in A}{\bigcup} B$ is an intuitionistic fuzzy semi-pre open set [13] and hence $A$ is an intuitionistic fuzzy generalized semi-pre open set in $X$. Thus $A \in \operatorname{IFGSPO}(X)$.

Definition 2.24. ([9]) A mapping $f:(X, \tau) \rightarrow(Y, \tau)$ is called an intuitionistic fuzzy generalized semi-pre continuous mapping (IFGSPCts. for short) if $f^{-1}(V)$ is an intuitionistic fuzzy generalized semi-pre closed set in $(X, \tau)$ for every intuitionistic fuzzy closed set $V$ of $(Y, \sigma)$.

\section{Intuitionistic fuzzy almost generalized semi-pre continuous mappings}

In this section we introduce intuitionistic fuzzy almost generalized semi-pre continuous mapping and investigate some of its properties.

Definition 3.1. A mapping $f: X \rightarrow Y$ is said to be an intuitionistic fuzzy almost generalized semi-pre continuous mapping (IFaGSPCts. for short) if $f^{-1}(A)$ is an intuitionistic fuzzy generalized semi-pre closed set in $X$ for every intuitionistic fuzzy regular closed set $A$ in $Y$.

For the sake of simplicity, we shall use the notation $A=\left\langle x,\left(\mu_{a}, \mu_{b}\right),\left(v_{a}, v_{b}\right)\right\rangle$ instead of $A=\left\langle x,\left(a / \mu_{a}, b / \mu_{b}\right),\left(a / v_{a}, b / v_{b}\right)\right\rangle$ in the following examples.

Similarly we shall use the notation $B=\left\langle y,\left(\mu_{u}, \mu_{v}\right),\left(v_{u}, v_{v}\right)\right\rangle$ instead of $B=\left\langle y,\left(u / \mu_{u}, v / \mu_{v}\right)\right.$, $\left.\left(u / v_{u}, v / v_{v}\right)\right\rangle$ in the following examples. 
Example 3.2. Let $X=\{a, b\}, Y=\{u, v\}$ and $G_{1}=\left\langle x,\left(0.5_{a}, 0.6_{b}\right),\left(0.5_{a}, 0.4_{b}\right)\right\rangle, G_{2}=\left\langle y,\left(0.2_{u}, 0.3_{v}\right)\right.$, $\left.\left(0.8_{u}, 0.7_{v}\right)\right\rangle$. Then $\tau=\left\{0_{\sim}, G_{1}, 1_{\sim}\right\}$ and $\sigma=\left\{0_{\sim}, G_{2}, 1_{\sim}\right\}$ are IFTs on $X$ and $Y$ respectively. Define a mapping $f:(X, \tau) \rightarrow(Y, \sigma)$ by $f(a)=u$ and $f(b)=v$. Then $f$ is an intuitionistic fuzzy almost generalized semi-pre continuous mapping.

Theorem 3.3. Every intuitionistic fuzzy continuous mapping is an intuitionistic fuzzy almost generalized semi-pre continuous mapping but not conversely.

Proof. Let $f:(X, \tau) \rightarrow(Y, \sigma)$ be an intuitionistic fuzzy continuous mapping. Let $V$ be an intuitionistic fuzzy regular closed set in $Y$. Since every intuitionistic fuzzy regular closed set is an intuitionistic fuzzy closed set, $V$ is an intuitionistic fuzzy closed set in $Y$. Then $f^{-1}(V)$ is an intuitionistic fuzzy closed set in $X$. Since every intuitionistic fuzzy closed set is an intuitionistic fuzzy generalized semi-pre closed set, $f^{-1}(V)$ is an intuitionistic fuzzy generalized semi-pre closed set in $X$. Hence $f$ is an intuitionistic fuzzy almost generalized semi-pre continuous mapping.

Example 3.4. Let $X=\{a, b\}, Y=\{u, v\}$ and $G_{1}=\left\langle x,\left(0.5_{a}, 0.4_{b}\right),\left(0.5_{a}, 0.6_{b}\right)\right\rangle, G_{2}=\left\langle x,\left(0.2_{a}, 0.3_{b}\right)\right.$, $\left.\left(0.8_{a}, 0.7_{b}\right)\right\rangle, G_{3}=\left\langle y,\left(0.2_{a}, 0.2_{b}\right),\left(0.4_{a}, 0.4_{b}\right)\right\rangle$ and $G_{4}=\left\langle y,\left(0.6_{u}, 0.6_{v}\right),\left(0.4_{u}, 0.4_{v}\right)\right\rangle$. Then $\tau=$ $\left\{0_{\sim}, G_{1}, G_{2}, 1_{\sim}\right\}$ and $\sigma=\left\{0_{\sim}, G_{3}, G_{4}, 1_{\sim}\right\}$ are IFTs on $X$ and $Y$ respectively. Define a mapping $f$ : $(X, \tau) \rightarrow(Y, \sigma)$ by $f(a)=u$ and $f(b)=v$. Then $f$ is an intuitionistic fuzzy almost generalized semi-pre continuous mapping but not anintuitionistic fuzzy continuous mapping, since $G_{4}^{c}$ is an intuitionistic fuzzy closed set in $Y$ but $f^{-1}\left(G_{4}^{c}\right)=\left\langle x,\left(0.4_{a}, 0.4_{b}\right),\left(0.6_{a}, 0.6_{b}\right)\right\rangle$ is not an intuitionistic fuzzy closed set in $X$.

Theorem 3.5. Every intuitionistic fuzzy generalized continuous mapping is an intuitionistic fuzzy almost generalized semi-pre continuous mapping but not conversely.

Proof. Let $f:(X, \tau) \rightarrow(Y, \sigma)$ be an intuitionistic fuzzy generalized continuous mapping. Let $V$ be an intuitionistic fuzzy regular closed set in $Y$. Since every intuitionistic fuzzy regular closed set is an intuitionistic fuzzy closed set, $V$ is an intuitionistic fuzzy closed set in $Y$. Then $f^{-1}(V)$ is an intuitionistic fuzzy generalized closed set in $X$. Since every intuitionistic fuzzy generalized closed set is an intuitionistic fuzzy generalized semi-pre closed set, $f^{-1}(V)$ is an intuitionistic fuzzy generalized semi-pre closed set in $X$. Hence $f$ is an intuitionistic fuzzy almost generalized semi-pre continuous mapping.

Example 3.6. In Example 3.4, $f$ is an intuitionistic fuzzy almost generalized semi-pre continuous mapping but not an intuitionistic fuzzy generalized continuous mapping, since $f^{-1}\left(G_{4}^{c}\right)=$ $\left\langle x,\left(0.4_{a}, 0.4_{b}\right),\left(0.6_{a}, 0.6_{b}\right)\right\rangle \subseteq G_{1}$ but $\operatorname{cl}\left(f^{-1}\left(G_{4}^{c}\right)\right)=G_{1}^{c} \nsubseteq G_{1}$.

Theorem 3.7. Every intuitionistic fuzzy semi continuous mapping is an intuitionistic fuzzy almost generalized semi-pre continuous mapping but not conversely. 
Proof. Let $f:(X, \tau) \rightarrow(Y, \sigma)$ be an intuitionistic fuzzy semi continuous mapping. Let $V$ be an intuitionistic fuzzy regular closed set in $Y$ Since every intuitionistic fuzzy regular closed set is an intuitionistic fuzzy closed set, $V$ is an intuitionistic fuzzy closed set in $Y$. Then $f^{-1}(V)$ is an intuitionistic fuzzy semi closed set in $X$. Since every intuitionistic fuzzy semi closed set is an intuitionistic fuzzy generalized semi-pre closed set, $f^{-1}(V)$ is an intuitionistic fuzzy generalized semi-pre closed set in $X$. Hence $f$ is an intuitionistic fuzzy almost generalized semi-pre continuous mapping.

Example 3.8. In Example 3.4, $f$ is an intuitionistic fuzzy almost generalized semi-pre continuous mapping but not an intuitionistic fuzzy semi continuous mapping, since $G_{4}^{c}$ is an intuitionistic fuzzy closed set in $Y$ but $f^{-1}\left(G_{4}^{c}\right)=\left\langle x,\left(0.4_{a}, 0.4_{b}\right),\left(0.6_{a}, 0.6_{b}\right)\right\rangle$ is not an intuitionistic fuzzy semi closed set in $Y$, since $\operatorname{int}\left(\operatorname{cl}\left(f^{-1}\left(G_{4}^{c}\right)\right)\right)=G_{1} \nsubseteq f^{-1}\left(G_{4}^{c}\right)$.

Theorem 3.9. Every intuitionistic fuzzy pre continuous mapping is an intuitionistic fuzzy almost generalized semi-pre continuous mapping but not conversely.

Proof. Let $f:(X, \tau) \rightarrow(Y, \sigma)$ be an intuitionistic fuzzy pre continuous mapping. Let $V$ be an intuitionistic fuzzy regular closed set in $Y$. Since every intuitionistic fuzzy regular closed set is an intuitionistic fuzzy closed set, $V$ is an intuitionistic fuzzy closed set in $Y$. Then $f^{-1}(V)$ is an intuitionistic fuzzy pre closed set in $X$. Since every intuitionistic fuzzy pre closed set is an intuitionistic fuzzy generalized semi-pre closed set, $f^{-1}(V)$ is an intuitionistic fuzzy generalized semi-pre closed set in $X$. Hence $f$ is an intuitionistic fuzzy almost generalized semi-pre continuous mapping.

Example 3.10. In Example 3.4, $f$ is an intuitionistic fuzzy almost generalized semi-pre continuous mapping but not an intuitionistic fuzzy pre continuous mapping, since $G_{4}^{c}$ is an intuitionistic fuzzy closed set in $Y$ but $f^{-1}\left(G_{4}^{c}\right)=\left\langle x,\left(0.4_{a}, 0.4_{b}\right),\left(0.6_{a}, 0.6_{b}\right)\right\rangle$ is not an intuitionistic fuzzy pre closed set in $Y$, since $\operatorname{cl}\left(\operatorname{int}\left(f^{-1}\left(G_{4}^{c}\right)\right)\right)=G_{1}^{c} \nsubseteq f^{-1}\left(G_{4}^{c}\right)$.

Theorem 3.11. Every intuitionistic fuzzy semi-pre continuous mapping is an intuitionistic fuzzy almost generalized semi-pre continuous mapping but not conversely.

Proof. Let $f:(X, \tau) \rightarrow(Y, \sigma)$ be an intuitionistic fuzzy semi-pre continuous mapping. Let $V$ be an intuitionistic fuzzy regular closed set in $Y$. Since every intuitionistic fuzzy regular closed set is an intuitionistic fuzzy closed set, $V$ is an intuitionistic fuzzy closed set in $Y$. Then $f^{-1}(V)$ is an intuitionistic fuzzy semi-pre closed set in $X$. Since every intuitionistic fuzzy semi-pre closed set is an intuitionistic fuzzy generalized semi-pre closed set, $f^{-1}(V)$ is an intuitionistic fuzzy generalized semi-pre closed set in $X$. Hence $f$ is an intuitionistic fuzzy almost generalized semi-pre continuous mapping. 
Example 3.12. In Example 3.4, $f$ is an intuitionistic fuzzy almost generalized semi-pre continuous mapping but not an intuitionistic fuzzy semi-pre continuous mapping, since $G_{4}^{c}$ is an intuitionistic fuzzy closed set in $Y$ but $f^{-1}\left(G_{4}^{c}\right)=\left\langle x,\left(0.4_{a}, 0.4_{b}\right),\left(0.6_{a}, 0.6_{b}\right)\right\rangle$ is not an intuitionistic fuzzy semi-pre closed set in $Y$, since int $\left(\operatorname{cl}\left(\operatorname{int}\left(f^{-1}\left(G_{4}^{c}\right)\right)\right)\right)=G_{1} \nsubseteq f^{-1}\left(G_{4}^{c}\right)$.

Theorem 3.13. Every intuitionistic fuzzy $\alpha$ continuous mapping is an intuitionistic fuzzy almost generalized semi-pre continuous mapping but not conversely.

Proof. Let $f:(X, \tau) \rightarrow(Y, \sigma)$ be an intuitionistic fuzzy $\alpha$ continuous mapping. Let $V$ be an intuitionistic fuzzy regular closed set in $Y$. Since every intuitionistic fuzzy regular closed set is an intuitionistic fuzzy closed set, $V$ is an intuitionistic fuzzy closed set in $Y$. Then $f^{-1}(V)$ is an intuitionistic fuzzy $\alpha$ closed set in $X$. Since every intuitionistic fuzzy $\alpha$ closed set is an intuitionistic fuzzy generalized semi-pre closed set, $f^{-1}(V)$ is an intuitionistic fuzzy generalized semi-pre closed set in $X$. Hence $f$ is an intuitionistic fuzzy almost generalized semi-pre continuous mapping.

Example 3.14. In Example 3.4, $f$ is an intuitionistic fuzzy almost generalized semi-pre continuous mapping but not an intuitionistic fuzzy $\alpha$ continuous mapping, since $G_{4}^{c}$ is an intuitionistic fuzzy closed set in $Y$ but $f^{-1}\left(G_{4}^{c}\right)=\left\langle x,\left(0.4_{a}, 0.4_{b}\right),\left(0.6_{a}, 0.6_{b}\right)\right\rangle$ is not an intuitionistic fuzzy $\alpha$ closed set in $Y$, since $\operatorname{cl}\left(\operatorname{int}\left(\operatorname{cl}\left(f^{-1}\left(G_{4}^{c}\right)\right)\right)\right)=G_{1}^{c} \nsubseteq f^{-1}\left(G_{4}^{c}\right)$.

Theorem 3.15. Let $f:(X, \tau) \rightarrow(Y, \sigma)$ be a mapping where $f^{-1}(V)$ is an intuitionistic fuzzy regular closed set in $X$ for every intuitionistic fuzzy closed set in $Y$. Then $f$ is an intuitionistic fuzzy almost generalized semi-pre continuous mapping but not conversely.

Proof. Let $A$ be an intuitionistic fuzzy regular closed set in $Y$. Since every intuitionistic fuzzy regular closed set is an intuitionistic fuzzy closed set, $V$ is an intuitionistic fuzzy closed set in $Y$. Then $f^{-1}(V)$ is an intuitionistic fuzzy regular closed set in $X$. Since every intuitionistic fuzzy regular closed set is an intuitionistic fuzzy generalized semi-pre closed set, $f^{-1}(V)$ is an intuitionistic fuzzy generalized semi-pre closed set in $X$. Hence $f$ is an intuitionistic fuzzy almost generalized semi-pre continuous mapping.

Example 3.16. In Example 3.4, $f$ is an intuitionistic fuzzy almost generalized semi-pre continuous mapping but not a mapping as defined in Theorem 3.15, since $G_{4}^{c}$ is an intuitionistic fuzzy closed set in $Y$ but $f^{-1}\left(G_{4}^{c}\right)=\left\langle x,\left(0.4_{a}, 0.4_{b}\right),\left(0.6_{a}, 0.6_{b}\right)\right\rangle$ is not an intuitionistic fuzzy regular closed set in $Y$, since $\operatorname{cl}\left(\operatorname{int}\left(f^{-1}\left(G_{4}^{c}\right)\right)\right)=G_{1} \neq f^{-1}\left(G_{4}^{c}\right)$.

Theorem 3.17. Every intuitionistic fuzzy generalized semi-pre continuous mapping is an intuitionistic fuzzy almost generalized semi-pre continuous mapping but not conversely. 
Proof. Let $f: X \rightarrow Y$ be an intuitionistic fuzzy generalized semi-pre continuous mapping. Let $A$ be an intuitionistic fuzzy regular closed set in $Y$. Then $A$ is an intuitionistic fuzzy closed set in $Y$. By hypothesis $f^{-1}(A)$ is an intuitionistic fuzzy generalized semi-pre closed set in $X$. Hence $f$ is an intuitionistic fuzzy almost generalized semi-pre continuous mapping.

Example 3.18. Let $X=\{a, b\}, Y=\{u, v\}$ and $G_{1}=\left\langle x,\left(0.5_{a}, 0.6_{b}\right),\left(0.2_{a}, 0_{b}\right)\right\rangle, G_{2}=\left\langle x,\left(0.4_{a}, 0.1_{b}\right)\right.$, $\left.\left(0.2_{a}, 0.1_{b}\right)\right\rangle, G_{3}=\left\langle y,\left(0.2_{u}, 0.2_{v}\right),\left(0.4_{u}, 0.4_{v}\right)\right\rangle$ and $G_{4}=\left\langle y,\left(0.2_{u}, 0_{v}\right),\left(0.5_{u}, 0.4_{v}\right)\right\rangle$. Then $\tau=\left\{0_{\sim}\right.$, $\left.G 1, G 2,1_{\sim}\right\}$ and $\sigma=\left\{0_{\sim}, G_{3}, G_{4}, 1_{\sim}\right\}$ are IFTs on $X$ and $Y$ respectively. Define a mapping $f$ : $(X, \tau) \rightarrow(Y, \sigma)$ by $f(a)=u$ and $f(b)=v$. Then $f$ is an intuitionistic fuzzy almost generalized semi-pre continuous mapping but not an intuitionistic fuzzy generalized semi-pre continuous mapping, since $f^{-1}\left(G_{4}\right)=\left\langle y,\left(0.2_{u}, 0_{v}\right),\left(0.5_{u}, 0.4_{v}\right)\right\rangle \subseteq G_{1}$ but $\operatorname{spcl}\left(f^{-1}\left(G_{4}\right)\right)=1 \sim \nsubseteq G_{1}$, is not an intuitionistic fuzzy generalized semi-pre closed set in $X$.

The relation between various types of intuitionistic fuzzy continuity is given in the following diagram. In this diagram Cts. means continuous.

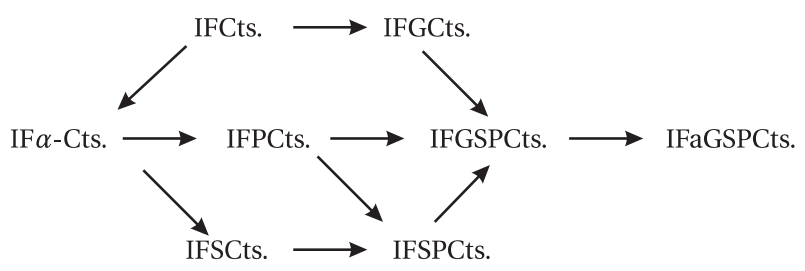

The reverse implications are not true in general in the above diagram.

Definition 3.19. An IFS $A$ is said to be an intuitionistic fuzzy dense (IFD for short) in another IFS $B$ in an IFTS $(X, \tau)$, if $\operatorname{cl}(A)=B$.

Theorem 3.20. Let $c(\alpha, \beta)$ be an intuitionistic fuzzy point in $X$. A mapping $f: X \rightarrow Y$ is an intuitionistic fuzzy almost generalized semi-pre continuous mapping if for every intuitionistic fuzzy open set $A$ in $Y$ with $f(c(\alpha, \beta)) \in A$, there exists an intuitionistic fuzzy open set $B$ in $X$ with $c(\alpha, \beta) \in B$ such that $f^{-1}(A)$ is IFD in $B$.

Proof. Let $A$ be an intuitionistic fuzzy regular open set in $Y$. Then $A$ is an intuitionistic fuzzy open set in $Y$. Let $f(c(\alpha, \beta)) \in A$, then there exists an intuitionistic fuzzy open set $B$ in $X$ such that $c(\alpha, \beta) \in B$ and $\operatorname{cl}\left(f^{-1}(A)\right)=B$. Since $B$ is an intuitionistic fuzzy open set, $\operatorname{cl}\left(f^{-1}(A)\right)=B$ is also an intuitionistic fuzzy open set in $X$. Therefore $\operatorname{int}\left(\operatorname{cl}\left(f^{-1}(A)\right)\right)=\operatorname{cl}\left(f^{-1}(A)\right)$. Now $f^{-1}(A) \subseteq \operatorname{cl}\left(f^{-1}(A)\right)=\operatorname{int}\left(\operatorname{cl}\left(f^{-1}(A)\right)\right) \subseteq \operatorname{cl}\left(\operatorname{int}\left(\operatorname{cl}\left(f^{-1}(A)\right)\right)\right)$. This implies $f^{-1}(A)$ is an intuitionistic fuzzy semi-pre open set in $X$ and hence an intuitionistic fuzzy generalized semi-pre open set in $X$. Thus $f$ is an intuitionistic fuzzy almost generalized semi-pre continuous mapping. 
Theorem 3.21. Let $f: X \rightarrow Y$ be a mapping where $X$ is an intuitionistic fuzzy semi-pre $T_{1 / 2}$ space. Then the following are equivalent

(i) $f$ is an intuitionistic fuzzy almost generalized semi-pre continuous mapping.

(ii) $\operatorname{spcl}\left(f^{-1}(A)\right) \subseteq f^{-1}(\operatorname{cl}(A))$ for every intuitionistic fuzzy semi-pre open set $A$ in $Y$.

(iii) $\operatorname{spcl}\left(f^{-1}(A)\right) \subseteq f^{-1}(\operatorname{cl}(A))$ for every intuitionistic fuzzy semi open set $A$ in $Y$.

(iv) $f^{-1}(A) \subseteq \operatorname{spint}\left(f^{-1}(\operatorname{int}(c l(A)))\right)$ for every intuitionistic fuzzy preopen set $A$ in $Y$.

Proof. (i) $\Rightarrow$ (ii) Let $A$ be an intuitionistic fuzzy semi-pre open set in $Y$. Then $\operatorname{cl}(A)$ is an intuitionistic fuzzy regular closed set in $Y$. By hypothesis $f^{-1}(A)$ is an intuitionistic fuzzy generalized semi-pre closed set in $X$ and hence is an intuitionistic fuzzy semi-pre closed set in $X$, since $X$ is an intuitionistic fuzzy semi-pre $T_{1 / 2}$ space. This implies $\operatorname{spcl}\left(f^{-1}(\operatorname{cl}(A))\right)=$ $f^{-1}(\operatorname{cl}(A))$. Now $\operatorname{spcl}\left(f^{-1}(A)\right) \subseteq \operatorname{spcl}\left(f^{-1}(\operatorname{cl}(A))\right)=f^{-1}(\operatorname{cl}(A))$. Thus $\operatorname{spcl}\left(f^{-1}(A)\right) \subseteq f^{-1}(\operatorname{cl}(A))$.

(ii) $\Rightarrow$ (iii) Since every intuitionistic fuzzy semi open set is an intuitionistic fuzzy semi-pre open set, proof is similar as in (i) $\Rightarrow$ (ii).

(iii) $\Rightarrow$ (i) Let $A$ be an intuitionistic fuzzy regular closed set in $Y$. Then $A=\operatorname{cl}(\operatorname{int}(A))$. Therefore $A$ is an intuitionistic fuzzy semi open set in $Y$. By hypothesis, $\operatorname{spcl}\left(f^{-1}(A)\right) \subseteq f^{-1}(\operatorname{cl}(A))=$ $f^{-1}(A) \subseteq \operatorname{spcl}\left(f^{-1}(A)\right)$. Hence $f^{-1}(A)$ is an intuitionistic fuzzy semi-pre closed set and hence is an intuitionistic fuzzy generalized semi-pre closed set in $X$. Thus $f$ is an intuitionistic fuzzy almost generalized semi-pre continuous mapping.

(i) $\Rightarrow$ (iv) Let $A$ be an intuitionistic fuzzy preopen set in $Y$. Then $A \subseteq \operatorname{int}(\operatorname{cl}(A))$. Since int $(\operatorname{cl}(A))$ is an intuitionistic fuzzy regular open set in $Y$, by hypothesis, $f^{-1}(\operatorname{int}(\operatorname{cl}(A)))$ is an intuitionistic fuzzy generalized semi-pre open set in $X$. Since $X$ is an intuitionistic fuzzy semi-pre $T_{1 / 2}$ space, $f^{-1}(\operatorname{int}(\operatorname{cl}(A)))$ is an intuitionistic fuzzy semi-pre open set in $X$. Therefore $f^{-1}(A) \subseteq$ $f^{-1}(\operatorname{int}(\operatorname{cl}(A))) \subseteq \operatorname{spint}\left(f^{-1}(\operatorname{int}(\operatorname{cl}(A)))\right)$.

(iv) $\Rightarrow$ (i) Let $A$ be an intuitionistic fuzzy regular open set in $Y$. Then $A$ is an intuitionistic fuzzy preopen set in $X$. By hypothesis, $f^{-1}(A) \subseteq \operatorname{spint}\left(f^{-1}(\operatorname{int}(\operatorname{cl}(A)))\right)=\operatorname{spint}\left(f^{-1}(A)\right) \subseteq f^{-1}(A)$. This implies $f^{-1}(A)$ is an intuitionistic fuzzy semi-pre open set in $X$ and hence is an intuitionistic fuzzy generalized semi-pre open set in $X$. Therefore $f$ is an intuitionistic fuzzy almost generalized semi-pre continuous mapping.

Theorem 3.22. Let $f: X \rightarrow Y$ be a mapping. Then the following are equivalent.

(i) $f$ is an intuitionistic fuzzy almost generalized semi-pre continuous mapping.

(ii) $f^{-1}(A)$ is an intuitionistic fuzzy generalized semi-pre open set in $X$ for every intuitionistic fuzzy regular open set $A$ in $Y$. 
Proof. (i) $\Rightarrow$ (ii) Let $A$ be an intuitionistic fuzzy regular closed set in $Y$. Then $A^{c}$ is an intuitionistic fuzzy regular open set in $Y$. By hypothesis, $f^{-1}\left(A^{c}\right)$ is an intuitionistic fuzzy generalized semipre open set in $X$. That is $f^{-1}(A)^{c}$ is an intuitionistic fuzzy generalized semi-pre open set in $X$. Therefore $f^{-1}(A)$ is an intuitionistic fuzzy generalized semi-pre closed set in $X$. Then $f$ is an intuitionistic fuzzy almost generalized semi-pre continuous mapping.

(ii) $\Rightarrow$ (i) Let $A$ be an intuitionistic fuzzy regular open set in $Y$. Then $A^{c}$ is an intuitionistic fuzzy regular closed set in $Y$. By hypothesis, $f^{-1}\left(A^{c}\right)$ is an intuitionistic fuzzy generalized semi-pre closed set in $X$. That is $f^{-1}(A)^{c}$ is an intuitionistic fuzzy generalized semi-pre closed set in $X$. Therefore $f^{-1}(A)$ is an intuitionistic fuzzy generalized semi-pre open set in $X$.

Theorem 3.23. Let $f: X \rightarrow Y$ be a mapping. Then $f$ is an intuitionistic fuzzy almost generalized semi-pre continuous mapping if for each intuitionistic fuzzy point $c(\alpha, \beta) \in X$ and for each intuitionistic fuzzy semi-pre open set $B$ in $Y$ such that $f(c(\alpha, \beta)) \in B, \operatorname{spcl}\left(f^{-1}(B)\right)$ is an IFSN of $c(\alpha, \beta) \in X$.

Proof. Let $c(\alpha, \beta) \in X$ and let $A$ be an intuitionistic fuzzy regular open set in $Y$. Then $A$ is an intuitionistic fuzzy semi-pre open set in $Y$. By hypothesis $f(c(\alpha, \beta)) \in A$, that is $c(\alpha, \beta) \in$ $f^{-1}(A)$ in $X$ and $\operatorname{spcl}\left(\left(f^{-1}(A)\right)\right.$ is an IFSN of $c(\alpha, \beta)$ in $X$. Therefore there exists an intuitionistic fuzzy semi-pre open set $B$ in $X$ such that $c(\alpha, \beta)) \in B \subseteq \operatorname{spcl}\left(f^{-1}(A)\right)$. We have $c(\alpha, \beta) \in$ $f^{-1}(A) \subseteq \operatorname{spcl}\left(f^{-1}(A)\right)$. Now $B=\bigcup\{c(\alpha, \beta) \mid c(\alpha, \beta) \in B\}=f^{-1}(A)$. Therefore $f^{-1}(A)$ is an intuitionistic fuzzy semi-pre open set in $X$ and hence an intuitionistic fuzzy generalized semi-pre open set in $X$. Thus $f$ is an intuitionistic fuzzy almost generalized semi-pre continuous mapping.

Theorem 3.24. Let $f: X \rightarrow Y$ be a mapping. If $f$ is an intuitionistic fuzzy almost generalized semi-pre continuous mapping, then $\operatorname{gspcl}\left(f^{-1}(A) \subseteq f^{-1}(\operatorname{cl}(A))\right.$ for every intuitionistic fuzzy semi-pre open set $A$ in $Y$.

Proof. Let $A$ be an intuitionistic fuzzy semi-pre open set in $Y$. Then $\operatorname{cl}(A)$ is an intuitionistic fuzzy regular closed set in $Y$. By hypothesis $f^{-1}(\operatorname{cl}(A))$ is an intuitionistic fuzzy generalized semi-pre closed set in $X$. Then $\operatorname{gspcl}\left(f^{-1}(\operatorname{cl}(A))=f^{-1}(\operatorname{cl}(A))\right.$. Now $\operatorname{gspcl}\left(f^{-1}(A)\right) \subseteq \operatorname{gspcl}\left(f^{-1}\right.$ $(\operatorname{cl}(A)))=f^{-1}(\operatorname{cl}(A))$. That is $\operatorname{gspcl}\left(f^{-1}(A) \subseteq f^{-1}(\operatorname{cl}(A))\right.$.

Corollary 3.25. Let $f: X \rightarrow Y$ be a mapping. If $f$ is an intuitionistic fuzzy almost generalized semi-pre continuous mapping, then $\operatorname{gspcl}\left(f^{-1}(A) \subseteq f^{-1}(\operatorname{cl}(A))\right.$ for every intuitionistic fuzzy semi open set A in $Y$.

Proof. Since every intuitionistic fuzzy semi open set is an intuitionistic fuzzy semi-pre open set, the proof directly follows from the Theorem 3.24 . 
Corollary 3.26. Let $f: X \rightarrow Y$ be a mapping. If $f$ is an intuitionistic fuzzy almost generalized semi-pre continuous mapping, then $\operatorname{gspcl}\left(f^{-1}(A) \subseteq f^{-1}(\operatorname{cl}(A))\right.$ for every intuitionistic fuzzy preopen set A in $Y$.

Proof. Since every intuitionistic fuzzy preopen set is an intuitionistic fuzzy semi-pre open set, the proof directly follows from the Theorem 3.24.

Theorem 3.27. Let $f: X \rightarrow Y$ be a mapping. If $f$ is an intuitionistic fuzzy almost generalized semi-pre continuous mapping, then $\operatorname{gspcl}\left(f^{-1}(\operatorname{cl}(A))\right) \subseteq f^{-1}(\operatorname{cl}(\operatorname{spint}(A)))$ for every intuitionistic fuzzy semi-pre open set A in $Y$.

Proof. Let $A$ be an intuitionistic fuzzy semi-pre open set in $Y$. Then $\operatorname{cl}(A)$ is an intuitionistic fuzzy regular closed set in $Y$. By hypothesis, $f^{-1}(\operatorname{cl}(A))$ is an intuitionistic fuzzy generalized semi-pre closed set in $X$. Then $\operatorname{gspcl}\left(f^{-1}(\operatorname{cl}(A))\right)=f^{-1}(\operatorname{cl}(A)) \subseteq f^{-1}(\operatorname{cl}(\operatorname{spint}(A)))$, since $\operatorname{spint}(A)=A$.

Corollary 3.28. Let $f: X \rightarrow Y$ be a mapping. If $f$ is an intuitionistic fuzzy almost generalized semi-pre continuous mapping, then $\operatorname{gspcl}\left(f^{-1}(\operatorname{cl}(A))\right) \subseteq f^{-1}(\operatorname{cl}(\operatorname{spint}(A)))$ for every intuitionistic fuzzy semi open set $A$ in $Y$.

Proof. Since every intuitionistic fuzzy semi open set is an intuitionistic fuzzy semi-pre open set, the proof directly follows from the Theorem 3.27.

Corollary 3.29. Let $f: X \rightarrow Y$ be a mapping. If $f$ is an intuitionistic fuzzy almost generalized semi-pre continuous mapping, then $\operatorname{gspcl}\left(f^{-1}(\operatorname{cl}(A))\right) \subseteq f^{-1}(\operatorname{cl}(\operatorname{spint}(A)))$ for every intuitionistic fuzzy preopen set $A$ in $Y$.

Proof. Since every intuitionistic fuzzy preopen set is an intuitionistic fuzzy semi-pre open set, the proof directly follows from the Theorem 3.27.

Theorem 3.30. Let $f: X \rightarrow Y$ be a mapping. If $f^{-1}(\operatorname{spint}(B)) \subseteq \operatorname{spint}\left(f^{-1}(B)\right)$ for every IFS $B$ in $Y$, then $f$ is an intuitionistic fuzzy almost generalized semi-pre continuous mapping.

Proof. Let $B \subseteq Y$ be an intuitionistic fuzzy regular open set. By hypothesis, $f^{-1}(\operatorname{spint}(B)) \subseteq$ $\operatorname{spint}\left(f^{-1}(B)\right)$. Since $B$ is an intuitionistic fuzzy regular open set, it is an intuitionistic fuzzy semi-pre open set in $Y$. Therefore $\operatorname{spint}(B)=B$. Hence $f^{-1}(B)=f^{-1}(\operatorname{spint}(B)) \operatorname{spint}\left(f^{-1}(B)\right) \subseteq$ $f^{-1}(B)$. This implies $f^{-1}(B)$ is an intuitionistic fuzzy semi-pre open set and hence an intuitionistic fuzzy generalized semi-pre open set in $X$. Thus $f$ is an intuitionistic fuzzy almost generalized semi-pre continuous mapping.

Remark 3.31. The converse of the above theorem is true if $B \subseteq Y$ is an intuitionistic fuzzy regular open set and $X$ is an intuitionistic fuzzy semi-pre $T_{1 / 2}$ space. 
Proof. Let $f$ be an intuitionistic fuzzy almost generalized semi-pre continuous mapping. Let $B$ be an intuitionistic fuzzy regular open set in $Y$. Then $f^{-1}(B)$ is an intuitionistic fuzzy generalized semi-pre open set in $X$. Since $X$ is an intuitionistic fuzzy semi-pre $T_{1 / 2}$ space, $f^{-1}(B)$ is an intuitionistic fuzzy semi-pre open set in $Y$. Therefore $f^{-1}(B)=f^{-1}(\operatorname{spint}(B)) \subseteq$ $\operatorname{spint}\left(f^{-1}(B)\right)$.

Theorem 3.32. Let $f: X \rightarrow Y$ be a mapping. If $\operatorname{spcl}\left(f^{-1}(B)\right) \subseteq f^{-1}(\operatorname{spcl}(B))$ for every IFS $B$ in $Y$, then $f$ is an intuitionistic fuzzy almost generalized semi-pre continuous mapping.

Proof. Let $B \subseteq Y$ be an intuitionistic fuzzy regular closed set. By hypothesis, $\operatorname{spcl}\left(f^{-1}(B)\right) \subseteq$ $f^{-1}(\operatorname{spcl}(B))$. Since $B$ is an intuitionistic fuzzy regular closed set, it is an intuitionistic fuzzy semi-pre closed set in $Y$. Therefore $\operatorname{spcl}(B)=B$. Hence $f^{-1}(B)=f^{-1}(\operatorname{spcl}(B)) \supseteq \operatorname{spcl}\left(f^{-1}(B)\right) \supseteq$ $f^{-1}(B)$. This implies $f^{-1}(B)$ is an intuitionistic fuzzy semi-pre closed set and hence an intuitionistic fuzzy generalized semi-pre closed set in $X$. Thus $f$ is an intuitionistic fuzzy almost generalized semi-pre continuous mapping.

Remark 3.33. The converse of the above theorem is true if $B \subseteq Y$ is an intuitionistic fuzzy regular closed set and $X$ is an intuitionistic fuzzy semi-pre $T_{1 / 2}$ space.

Proof. Let $f$ be an intuitionistic fuzzy almost generalized semi-pre continuous mapping. Let $B$ be an intuitionistic fuzzy regular closed set in $Y$. Then $f^{-1}(B)$ is an intuitionistic fuzzy generalized semi-pre closed set in $X$. Since $X$ is an intuitionistic fuzzy semi-pre $T_{1 / 2}$ space, $f^{-1}(B)$ is an intuitionistic fuzzy rsemi-pre closed set in $Y$. Therefore $\left.\operatorname{spcl}\left(f^{-1}(B)\right)=f^{-1}(B)\right) \subseteq$ $f^{-1}(\operatorname{spcl}(B))$.

Theorem 3.34. Let $f: X \rightarrow Y$ be a mapping where $X$ is an intuitionistic fuzzy semi-pre $T_{1 / 2}$ space. Then the following are equivalent.

(i) $f$ is an intuitionistic fuzzy almost generalized semi-pre continuous mapping.

(ii) for each intuitionistic fuzzy point $c(\alpha, \beta)$ in $X$ and each intuitionistic fuzzy regular open set $B$ in $Y$ such that $f(c(\alpha, \beta)) \in B, \operatorname{cl}\left(f^{-1}(\operatorname{cl}(B))\right)$ is an IFSN of $c(\alpha, \beta)$ in $X$.

Proof. (i) $\Rightarrow$ (ii) Let $c(\alpha, \beta) \in X$ and let $B$ be an intuitionistic fuzzy regular open set in $Y$ such that $f(c(\alpha, \beta)) \in B$. That is $c(\alpha, \beta) \in f^{-1}(B)$. By hypothesis, $f^{-1}(B)$ is an intuitionistic fuzzy generalized semi-pre open set in $X$. Since $X$ is an intuitionistic fuzzy semi-pre $T_{1 / 2}$ space, $f^{-1}(B)$ is an intuitionistic fuzzy semi-pre open set in $X$. Now $c(\alpha, \beta) \in f^{-1}(B) \subseteq f^{-1}(\operatorname{cl}(B)) \subseteq$ $\operatorname{cl}\left(f^{-1}(\operatorname{cl}(B))\right)$. Hence $\operatorname{cl}\left(f^{-1}(\operatorname{cl}(B))\right)$ is an IFSN of $c(\alpha, \beta)$ in $X$.

(ii) $\Rightarrow$ (i) Let $B$ be an intuitionistic fuzzy open set in $Y$. Then $f(c(\alpha, \beta)) \in B$. This implies $c(\alpha, \beta) \in f^{-1}(B)$. By hypothesis, $\operatorname{cl}\left(f^{-1}(\operatorname{cl}(B))\right)$ is an IFSN of $c(\alpha, \beta)$. Therefore there exists an 
intuitionistic fuzzy semi-pre open set $A$ in $X$ such that $c(\alpha, \beta) \in A \subseteq \operatorname{cl}\left(f^{-1}(\operatorname{cl}(B))\right)$. Now $A=$ $\bigcup\{c(\alpha, \beta) \mid c(\alpha, \beta) \in A\}=f^{-1}(B)$. Therefore $f^{-1}(B)$ is an intuitionistic fuzzy semi-pre open set and hence an intuitionistic fuzzy generalized semipre open set in $X$. Thus $f$ is an intuitionistic fuzzy almost generalized semi-pre continuous mapping.

Theorem 3.35. The following are equivalent for a mapping $f: X \rightarrow Y$ where $X$ is an intuitionistic fuzzy semi-pre $T_{1 / 2}$ space.

(i) $f$ is an intuitionistic fuzzy almost generalized semi-pre continuous mapping.

(ii) $\operatorname{spcl}\left(f^{-1}(A)\right) \subseteq f^{-1}(\alpha c l(A))$ for every intuitionistic fuzzy semi-pre open set $A$ in $Y$.

(iii) $\operatorname{spcl}\left(f^{-1}(A)\right) \subseteq f^{-1}(\alpha c l(A))$ for every intuitionistic fuzzy semi open set $A$ in $Y$.

(iv) $f^{-1}(A) \subseteq \operatorname{spint}\left(f^{-1}(\operatorname{scl}(A))\right)$ for every intuitionistic fuzzy preopen set $A$ in $Y$.

Proof. (i) $\Rightarrow$ (ii) Let $A$ be an intuitionistic fuzzy semi-pre open set in $Y$. Then $\operatorname{cl}(A)$ is an intuitionistic fuzzy regular closed set in $Y$. By hypothesis $f^{-1}(A)$ is an intuitionistic fuzzy generalized semi-pre closed set in $X$ and hence is an intuitionistic fuzzy semi-pre closed set in $X$, since $X$ is an intuitionistic fuzzy semi-pre $T_{1 / 2}$ space. This implies $\operatorname{spcl}\left(f^{-1}(\operatorname{cl}(A))\right)=$ $f^{-1}(\operatorname{cl}(A))$. Now $\operatorname{spcl}\left(f^{-1}(A)\right) \subseteq \operatorname{spcl}\left(f^{-1}(\operatorname{cl}(A))\right)=f^{-1}(\operatorname{cl}(A))$. Since $\operatorname{cl}(A)$ is an intuitionistic fuzzy regular closed set, $\operatorname{cl}(\operatorname{int}(\operatorname{cl}(A)))=\operatorname{cl}(A)$. Now $\operatorname{spcl}\left(f^{-1}(A)\right) \subseteq f^{-1}(\operatorname{cl}(A))=f^{-1}(\operatorname{cl}(\operatorname{int}(\operatorname{cl}(A)$ )$) \subseteq f^{-1}(A \cup \operatorname{cl}(\operatorname{int}(\operatorname{cl}(A)))) \subseteq f^{-1}(\alpha \operatorname{cl}(A))$. Hence $\operatorname{spcl}\left(f^{-1}(A)\right) \subseteq f^{-1}(\alpha \operatorname{cl}(A))$.

(ii) $\Rightarrow$ (iii) Let $A$ be an intuitionistic fuzzy semi open set in $Y$. Since every intuitionistic fuzzy semi open set is an intuitionistic fuzzy semi-pre open set, the proof is obvious.

(iii) $\Rightarrow$ (i) Let $A$ be an intuitionistic fuzzy regular closed set in $Y$. Then $A=\operatorname{cl}(\operatorname{int}(A))$. Therefore $A$ is an intuitionistic fuzzy semi open set in $Y$. By hypothesis, $\operatorname{spcl}\left(f^{-1}(A)\right) \subseteq f^{-1}(\alpha \operatorname{cl}(A)) \subseteq$ $f^{-1}(\operatorname{cl}(A))=f^{-1}(A) \subseteq \operatorname{spcl}\left(f^{-1}(A)\right)$. That is $\operatorname{spcl}\left(f^{-1}(A)\right)=f^{-1}(A)$. Hence $f^{-1}(A)$ is an intuitionistic fuzzy semi-pre closed set and hence is an intuitionistic fuzzy generalized semi-pre closed set in $X$. Thus $f$ is an intuitionistic fuzzy almost generalized semi-pre continuous mapping.

(i) $\Rightarrow$ (iv) Let $A$ be an intuitionistic fuzzy preopen set in $Y$. Then $A \subseteq \operatorname{int}(\operatorname{cl}(A))$. Since int $(\operatorname{cl}(A))$ is an intuitionistic fuzzy regular open set in $Y$, by hypothesis, $f^{-1}(\operatorname{int}(\operatorname{cl}(A)))$ is an intuitionistic fuzzy generalized semi-pre open set in $X$. Since $X$ is an intuitionistic fuzzy semi-pre $T_{1 / 2}$ space, $f^{-1}(\operatorname{int}(\operatorname{cl}(A)))$ is an intuitionistic fuzzy semi-pre open set in $X$. Therefore $f^{-1}(A) \subseteq$ $f^{-1}(\operatorname{int}(\operatorname{cl}(A))) \subseteq \operatorname{spint}\left(f^{-1}(\operatorname{int}(\operatorname{cl}(A)))\right)=\operatorname{spint}\left(f^{-1}(A \cup \operatorname{int}(\operatorname{cl}(A)))\right)=\operatorname{spint}\left(f^{-1}(\operatorname{scl}(A))\right)$. That is $f^{-1}(A) \subseteq \operatorname{spint}\left(f^{-1}(\operatorname{scl}(A))\right)$.

(iv) $\Rightarrow$ (i) Let $A$ be an intuitionistic fuzzy regular open set in $Y$. Then $A$ is an intuitionistic fuzzy preopen set in $Y$. By hypothesis, $f^{-1}(A) \subseteq \operatorname{spint}\left(f^{-1}(\operatorname{scl}(A))\right)$. This implies $f^{-1}(A) \subseteq \operatorname{spint}\left(f^{-1}(A\right.$ 
$\bigcup \operatorname{lint}(\operatorname{cl}(A))))=\operatorname{spint}\left(f^{-1}(A \cup A)\right)=\operatorname{spint}\left(f^{-1}(A)\right) \subseteq f^{-1}(A)$. Therefore $f^{-1}(A)$ is an intuitionistic fuzzy semi-pre open set in $X$ and hence an intuitionistic fuzzy generalized semi-pre open set in $X$. Thus $f$ is an intuitionistic fuzzy almost generalized semi-pre continuous mapping.

Theorem 3.36. Let $f: X \rightarrow Y$ be a mapping where $X$ is an intuitionistic fuzzy semi-pre $T_{1 / 2}$ space. If $f$ is an intuitionistic fuzzy almost generalized semi-pre continuous mapping, then $\operatorname{int}\left(\operatorname{cl}\left(\operatorname{int}\left(f^{-1}(B)\right)\right)\right) \subseteq f^{-1}(\operatorname{spcl}(B))$ for every intuitionistic fuzzy regular closed set $B$ in $Y$.

Proof. Let $B \subseteq Y$ be an intuitionistic fuzzy regular closed set. By hypothesis, $f^{-1}(B)$ is an intuitionistic fuzzy generalized semi-pre closed set in $X$. Since $X$ is an intuitionistic fuzzy semi-pre $T_{1 / 2}$ space, $f^{-1}(B)$ is an intuitionistic fuzzy semi-pre closed set in $X$. Therefore $\operatorname{spcl}\left(f^{-1}(B)\right)=f^{-1}(B)$. Now $\operatorname{int}\left(\operatorname{cl}\left(\operatorname{int}\left(f^{-1}(B)\right)\right)\right) \subseteq f^{-1}(B) \bigcup \operatorname{int}\left(\operatorname{cl}\left(\operatorname{int}\left(f^{-1}(B)\right)\right)\right)=\operatorname{spcl}\left(f^{-1}(B)\right)$ $=f^{-1}(B)=f^{-1}(\operatorname{spcl}(B))$. Hence $\operatorname{int}\left(\operatorname{cl}\left(\operatorname{int}\left(f^{-1}(B)\right)\right)\right) \subseteq f^{-1}(\operatorname{spcl}(B))$.

Theorem 3.37. Let $f: X \rightarrow Y$ be a mapping where $X$ is an intuitionistic fuzzy semi-pre $T_{1 / 2}$ space. If $f$ is an intuitionistic fuzzy almost generalized semi-pre continuous mapping, then $f^{-1}(\operatorname{spint}(B)) \operatorname{cl}\left(\operatorname{int}\left(c l\left(f^{-1}(B)\right)\right)\right)$ for every intuitionistic fuzzy regular open set $B$ in $Y$.

Proof. This theorem can be easily proved by taking complement in Theorem 3.36.

\section{References}

[1] B. Ahmad and M. Athar, Fuzzy almost continuous functions, Int. J. Contemp. Math. Sciences, (2008), 16651677.

[2] K. Atanassov, Intuitionistic fuzzy sets, Fuzzy Sets and Systems, (1986), 87-96.

[3] Biljana Krsteska and Erdal Ekici, Intuitionistic fuzzy contra strong precontinuity, Faculty of Sciences and Mathematics, University of Nis, Serbia, (2007), 273-284.

[4] C. Chang, Fuzzy topological spaces, J. Math. Anal. Appl., (1968), 182-190.

[5] D. Coker, An introduction to intuitionistic fuzzy topological space, Fuzzy sets and Systems, (1997), 81-89.

[6] H. Gurcay, D. Coker and Es. A. Haydar, On fuzzy continuity in Intuitionistic fuzzy topological spaces, The J. fuzzy mathematics, (1997), 365-378.

[7] Joung Kon Jeon, Young Bae Jun and Jin Han Park, Intuitionistic fuzzy alpha-continuity and intuitionistic fuzzy pre continuity, International Journal of Mathematics and Mathematical Sciences, (2005), 3091-3101.

[8] R. Santhi and D. Jayanthi, Intuitionistic fuzzy generalized semi-pre closed sets (accepted).

[9] R. Santhi and D. Jayanthi, Intuitionistic fuzzy generalized semi-pre continuous mappings (submitted).

[10] Seok Jong Lee and Eun Pyo Lee, The category of intuitionistic fuzzy topological spaces, Bull. Korean Math. Soc. (2000), 63-76.

[11] S. S. Thakur and Rekha Chaturvedi, Regular generalized closed sets in intuitionistic fuzzy topological spaces, Universitatea Din Bacau, Studii Si Cercetari Stiintifice, Seria: Matematica, (2006), 257-272.

[12] Young Bae Jun and Seok-Zun Song, Intuitionistic fuzzy semi-pre open sets and Intuitionistic semi-pre continuous mappings, J. Appl. Math \& Computing, (2005), 467-474.

[13] L. A. Zadeh, Fuzzy sets, Information and Control, (1965), 338-353. 
Department of Mathematics, NGM College, Pollachi, Tamil Nadu.

E-mail: jayanthimaths@rediffmail.com

Department of Mathematics, NGM College, Pollachi, Tamil Nadu.

E-mail: santhifuzzy@yahoo.co.in 